\title{
Neprilysin Deficiency-Dependent Impairment of Cognitive Functions in a Mouse Model of Amyloidosis
}

\section{Journal Article}

Author(s):

Mohajeri, M. Hasan; Wolfer, David Paul (D)

Publication date:

2009

Permanent link:

https://doi.org/10.3929/ethz-b-000156668

Rights / license:

In Copyright - Non-Commercial Use Permitted

Originally published in:

Neurochemical Research 34(4), https://doi.org/10.1007/s11064-009-9919-6 


\title{
Neprilysin Deficiency-Dependent Impairment of Cognitive Functions in a Mouse Model of Amyloidosis
}

\author{
M. Hasan Mohajeri · David P. Wolfer
}

Accepted: 3 July 2008/Published online: 6 February 2009

(C) Springer Science+Business Media, LLC 2009

\begin{abstract}
Alzheimer's disease, responsible for the vast majority of dementia cases in the elderly population, is caused by accumulation of toxic levels of amyloid $\beta$ peptide $(\mathrm{A} \beta)$ in the brain. Neprilysin is a major enzyme responsible for the degradation of $\mathrm{A} \beta$ in vivo. We have previously shown that elevation of neprilysin levels in the brain delays the deposition of $\mathrm{A} \beta$-plaques in a mouse model of amyloidosis and that lack of neprilysin leads to increased $\mathrm{A} \beta$ generation and to signs of incipient neurodegeneration in mouse brains. This study was designed to test whether low brain levels of neprilysin affect the amyloid pathology or perturb the learning and memory performance of mice. Double-mutated mice carrying a targeted depletion of one allele of Mme, the gene encoding neprilysin, and over-expressing human amyloid precursor protein (APP), exhibited a reinforced amyloid pathology in comparison with their APP transgenic littermates. Moreover, in contrast to their parental lines, these mice were impaired in the Morris water maze learning and memory paradigm and showed facilitated extinction in the
\end{abstract}

Special issue to Honor Dr. Akitane Mori.

M. H. Mohajeri

Division of Psychiatry Research, University of Zurich,

Zurich, Switzerland

\section{P. Wolfer}

Institute of Anatomy, University of Zurich, Switzerland and Institute for Human Movement Sciences, ETH Zurich, Switzerland

\section{H. Mohajeri $(\bowtie)$}

DSM Nutritional Products Ltd., R\&D Human Nutrition and Health, Bldg. 205/217, P.O. Box 2676, CH-4002 Basel, Switzerland

e-mail: hasan.mohajeri@dsm.com conditioned taste aversion test. These data suggest that even a partial neprilysin deficiency, as is found during aging, exacerbates amyloid pathology and may impair cognitive functions.

Keywords Alzheimer $\cdot \mathrm{A} \beta \cdot$ Cognition · Therapy · Amyloid pathology $\cdot$ APP

\section{Introduction}

Accumulation of $\mathrm{A} \beta$ in the brain is viewed as the primary cause of Alzheimer's disease (AD) pathogenesis. $\mathrm{A} \beta$ accumulation is proposed to result from an imbalance between $\mathrm{A} \beta$ production and clearance and to induce the formation of neurofibrillary tangles [1]. The hippocampal formation is one of the first areas affected by $\mathrm{AD}$ and hippocampal CA1 and CA3 neurons are instrumental for learning and memory in rodents, nonhuman primates, and humans [2-5]. The finding that familial AD is caused by mutations in genes encoding the amyloid precursor protein (APP) and presenilins, leading to high production of the $\mathrm{A} \beta$ peptides in the brain, has established the importance of over-production of $\mathrm{A} \beta$ in $\mathrm{AD}$ pathophysiology. Consequently, mouse models were generated that produce $\mathrm{A} \beta$ pathology in an age-dependent manner. In contrast, a higher production rate of $\mathrm{A} \beta$ has not been found in sporadic $\mathrm{AD}$, which comprises more than $90 \%$ of $\mathrm{AD}$ cases. Therefore, the cause of accumulation of $\mathrm{A} \beta$ in sporadic cases is likely to be a deceleration of $\mathrm{A} \beta$ degradation $[6,7]$.

Neprilysin, a member of the metalloprotease family, was identified as the major physiological $\mathrm{A} \beta$-degrading enzyme in the brain $[8,9]$. Neprilysin levels in AD brain regions with high plaque content have been shown to be approximately half high as the levels found in control brain 
areas with low or moderate plaque burden $[10,11]$ and furthermore correlated inversely with amyloid load in mouse brains [12]. Mechanistically, the presence of neprilysin at presynaptic sites was shown to be of particular importance in protecting synapses from $\mathrm{A} \beta$-derived synaptotoxicity [13]. We have recently demonstrated that higher brain neprilysin activity delayed the deposition of amyloid plaques in a mouse model of amyloidosis, whereas, neprilysin deficiency led to $\mathrm{A} \beta$ accumulation and amyloid deposition in mouse brains $[14,15]$.

The objectives of this study were to determine whether low brain levels of neprilysin affect $\mathrm{A} \beta$ concentrations and amyloid plaque pathology in the brains of transgenic mice over-expressing human $\mathrm{A} \beta$, and whether altered amyloid pathology reduces the performance of these mice in behavioral tests. We report here that even a reduction of brain neprilysin activity to half of normal levels induced an accelerated accumulation of $\mathrm{A} \beta$ in mouse brains associated with significant impairments in hippocampal-dependent and independent cognitive tasks.

\section{Experimental Procedures}

Mice

Both parental mouse lines, with targeted depletion of the neprilysin gene [16] or the J20 mouse line with transgenic neuronal over-expression of human APP (APPtg) [17], were crossbred for more than 20 generations with the C57BL/6 background. Mice were housed under a light cycle of $12 \mathrm{~h}$, with free access to dry food pellets and water, and were weaned at 3 weeks of age. Cross-breeding of the mouse lines was performed by breeding male APPtg mice with female mice heterozygous for neprilysin depletion to generate wildtype (WT), APPtg, mice heterozygous for neprilysin deficiency (NEP-) and double-mutated mice heterozygote for neprilysin deficiency and over-expressing human APP (NEP-APPtg). The presence of the neprilysin knock-out versus WT allele and the APP transgene were determined as previously described $[14,17,18]$.

First mouse groups were tested in the open field and Morris water maze (MWM) behavioral tests, and then were analyzed by biochemical and immunohistochemical methods. A second group of mice was tested in the conditioned taste aversion (CTA) test. All experiments were performed following national and cantonal regulations and procedures as approved by the cantonal ethics committee.

\section{Behavioral Studies}

A total of 482 year-old mice ( $n=9-14$ per genotype), including balanced numbers of male and female subjects, were subjected to first round of behavioral testing. After assessing activity in an open field arena, mice were subjected to the MWM test, which measures hippocampusdependent spatial learning and memory. Finally, a second group of mice, originating from the same breeding pairs $(n=10$ per genotype, containing male and female mice, $24 \pm 1$ months of age), were subjected to the CTA test.

\section{Morris Water Maze}

A modified MWM protocol was employed as described previously [2]. Briefly, training and testing of mice were performed under 12 lux diffuse light in a circular pool constructed from white polypropylene (diameter $150 \mathrm{~cm}$, wall height $50 \mathrm{~cm}$ ), filled with opaque water to a height of $16 \mathrm{~cm}$ and maintained at $24-26^{\circ} \mathrm{C}$. In the cued or visible platform learning, mice were trained for 2 days (six trials per day) to find a $16 \mathrm{~cm} \times 16 \mathrm{~cm}$ target platform of white wire mesh, which was placed $0.5 \mathrm{~cm}$ above the surface of water and the position of which was changed with each trial. Subsequently, to examine the spatial learning, the platform was submerged $0.5 \mathrm{~cm}$ below the water surface in the NW, NE, SE or SW quadrant $35 \mathrm{~cm}$ from the pool wall. Each mouse was released to the pool from different starting points and was trained to locate the platform, which was maintained in the same position for an individual mouse, over 4 days with six trials per day separated by $30-60 \mathrm{~min}$ intervals and each trial lasting maximally $120 \mathrm{~s}$. About $24 \mathrm{~h}$ after completion of training, mice were subjected to a probe trial of $30 \mathrm{~s}$ without the goal platform to test spatial retention. Animals were tracked using a Noldus EthoVision 3.0 video-tracking system (www.noldus.com) at $576 \times 768$ pixels spatial resolution and $4.167 \mathrm{~Hz}$ sampling frequency. Raw XY coordinates were transferred to custom-developed public domain software Wintrack [21] (www.dpwolfer.ch/wintrack) for further analysis. Spatial selectivity during the probe trial was assessed using: (1) percentage time spent in a circular zone centered on the trained goal and comprising one-eighth of the pool surface (target zone) versus control zones in the other quadrants, and (2) the number of crossings over the target area and respective control annuli of $16 \mathrm{~cm}$ diameter.

\section{Conditioned Taste Aversion Test (CTA)}

Water-deprived mice ( $n=10$ per genotype) were housed individually and were trained over four consecutive days to drink from two drinking tubes for $20 \mathrm{~min}$ in the morning and $10 \mathrm{~min}$ in the afternoon, with a $4 \mathrm{~h}$ inter-trial interval $[14,18]$. The fluid intake for each mouse was determined by weighing the drinking tubes before and after each trial. On the conditioning day, mice were allowed to drink for 20 min from only one tube, which was filled with a $0.5 \%$ 
saccharin solution (Fluka, Buchs, Switzerland) (conditioned stimulus). Mice were then administered a $0.14 \mathrm{M}$ $\mathrm{LiCl}$ solution (at $0.2 \%$ of body weight, ip) as the nauseainducing agent (unconditioned stimulus) $40 \mathrm{~min}$ later. About $48 \mathrm{~h}$ after the conditioning trial, animals were subjected to the first preference choice test (retrieval trial), which constituted presentation of two drinking tubes, one containing $0.5 \%$ saccharin and one containing water (choice test) for $20 \mathrm{~min}$. The aversion coefficient was calculated as the percentage of saccharin consumption compared with the total fluid intake. This parameter correlates inversely with conditioned learning and memory performance. This test was repeated once per week over four consecutive weeks. Finally, the normal taste sensitivity of mouse groups was determined by testing the natural aversion towards a bitter taste by offering the mice $0.02 \%$ quinine solution (Fluka, Buchs, Switzerland) or water $[22,23]$.

\section{Biochemical Analysis}

Following behavioral testing, 7-8 mice per genotype were randomly chosen for biochemical analysis of brain tissue. Mice were perfused transcardially with ice cold PBS, whole brains were removed, followed by removal of the cerebellum and olfactory bulbs. Brains were first cut sagittally and then at bregma level dividing each hemisphere into a frontal region and the caudal brain tissue containing the hippocampus. The frontal parts and one hippocampuscontaining sample derived from each brain were frozen immediately. The remaining caudal sample of each brain was fixed for $48 \mathrm{~h}$ in $4 \%$ paraformaldehyde at $4^{\circ} \mathrm{C}$, washed several times in PBS and embedded in paraffin. The presence of amyloid plaques was determined in coronal sections including the hippocampus at interaural level $-1.5 \mathrm{~mm}$ to bregma, utilizing the $4 \mathrm{G} 8$ monoclonal antibody (Seretec) recognizing both human and murine $\mathrm{A} \beta$ as described [19]. The second sample of caudal brain tissue, containing the other hippocampus, was weighed and placed in ice-cold buffer $(1: 15 \mathrm{w} / \mathrm{v})$ containing $100 \mathrm{mM}$ Tris $(\mathrm{pH}$ 8.0), $150 \mathrm{mM} \mathrm{NaCl}, 2 \% \mathrm{SDS}$ and complete protease inhibitor cocktail (Roche), then homogenized at 1,000 rpm using a Potter $\mathrm{S}$ homogenizer. Brain homogenates were then centrifuged at $100,000 \mathrm{~g}$ for $1 \mathrm{~h}$ at $4^{\circ} \mathrm{C}$. The supernatant was collected and the remaining pellet was dissolved in $70 \%$ formic acid $(1: 3 \mathrm{w} / \mathrm{v})$. The concentrations of $\mathrm{A} \beta 40$ and $\mathrm{A} \beta 42$ were measured in the brain homogenates using commercially available kits measuring both human and murine A $\beta$ species (Wako Pure Chemical Industries, Ltd., Osaka, Japan) according to the manufacturer's instructions.

In addition, neprilysin enzymatic activity was measured in the frontal brain tissues, following homogenization in a buffer containing $100 \mathrm{mM}$ Tris (pH 8.0) and $150 \mathrm{mM}$
$\mathrm{NaCl}$, according to published methods $[19,20]$. Background absorbance readings were established using brain homogenates $(n=4)$ prepared from age-matched neprilysin knock-out mice [16].

\section{Statistical Analysis}

Data were collected by colleagues unaware of the genotypes of the mice. For behavioral tests, groups were always trained and tested in parallel and identically. Statistical analysis of biochemical and CTA data was performed using one way analysis of variance (ANOVA) and significant main effects of genotype were further analyzed using Fisher's PLSD posthoc test. For analysis of MWM performance, the data of all groups including males and females, were analyzed using a 3-way factorial ANOVA model with the between-subject factors of APPtg+ (APPtg vs. WT), neprilysin heterozygosity (NEP - vs. WT) and sex. No gender-dependent effects were found. Therefore, final analysis was performed using a two-way ANOVA without the gender factor. In order to fit the factorial ANOVA model, training variables were averaged over all training trials. For selected variables, the ANOVA model was complemented by the within-subject factors of time (blocks of two subsequent trials) and place (trained vs. average of non-trained sites), in order to assess learning rates and place preferences. To further explore potential interactions between the mutations, the ANOVA model was split along the APPtg + or NEP - factor. Data are expressed as mean \pm SEM, ns shows a non-significant effect.

\section{Results}

Neprilysin Deficiency was Associated with Increased Amyloid Pathology

Amyloid plaque load was studied in mouse brains via immunohistochemistry utilizing the 4G8 antibody, which recognizes both human and mouse $\mathrm{A} \beta$ peptides. APPtg mice exhibited small punctated and compacted amyloid plaques, whereas, the number and size of amyloid plaques were greater in the brains of doubly mutated NEP-APPtg mice (Fig. 1). Concentrations of SDS- and formic acid (FA)-soluble $\mathrm{A} \beta 40$ and $\mathrm{A} \beta 42$ were further quantified in each mouse brain (Table 1). In SDS homogenates of WT brains, the $\mathrm{A} \beta 40$ concentration was $0.69 \pm 0.05 \mathrm{nM}$, whereas, $\mathrm{A} \beta 42$ values did not exceed background levels. $\mathrm{A} \beta 40$ concentrations differed markedly between groups in the following manner: WT $<\mathrm{NEP}-<$ APPtg $<$ NEPAPPtg (Table 1). In the FA homogenates, however, measurable $\mathrm{A} \beta 40$ was only found in APPtg and NEPAPPtg brains with levels in the latter genotype being 
Fig. 1 Neprilysin deficiency exacerbates amyloid pathology in mouse brains. Frontal brain sections through the hippocampal formation were stained with the 4G8 antibody, which recognizes both human and murine $\mathrm{A} \beta$. APPtg mice exhibited distinct amyloid deposits but the amyloid plaque load was highest in doublymutated mice (NEP-APPtg) combining chronic low neprilysin levels and expression of the AD-associated human APP transgene
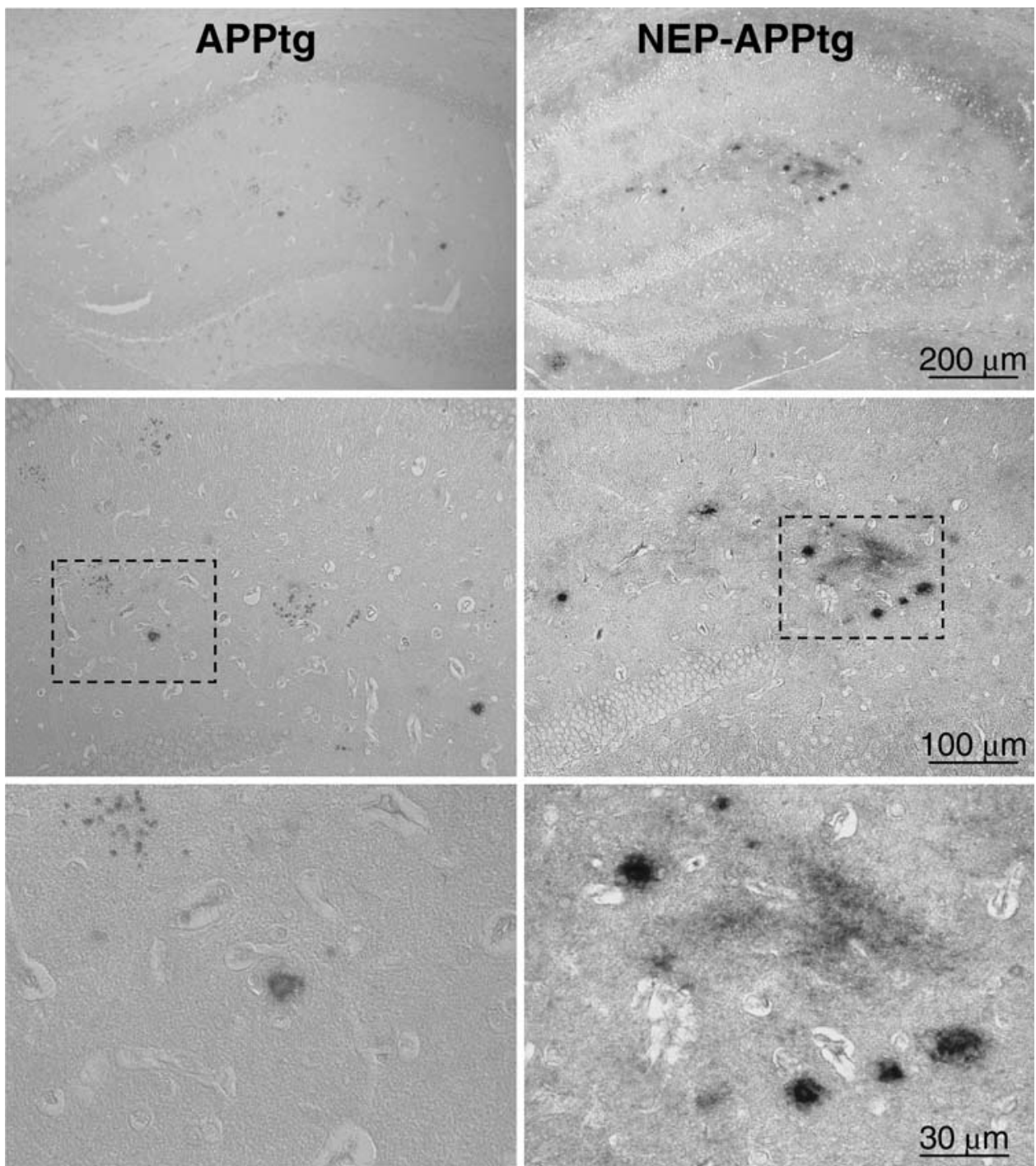

Table 1 Quantification of brain $\mathrm{A} \beta$ concentrations

\begin{tabular}{clllr}
\hline & WT & NEP- & APPtg & NEP-APPtg \\
\hline SDS soluble & & & & \\
$\mathrm{A} \beta 40(\mathrm{nM} \pm \mathrm{SEM})$ & $0.69 \pm 0.05$ & $1.27 \pm 0.09$ & $2.07 \pm 0.1$ & $\mathrm{Nd}$ \\
$\mathrm{A} \beta 42(\mathrm{pM} \pm \mathrm{SEM})$ & $\mathrm{Nd}$ & $\mathrm{Nd}$ & & $1,067 \pm 161$ \\
Formic acid soluble & & & $0.383 \pm 0.026$ & $1.049 \pm 0.10$ \\
$\mathrm{~A} \beta 40(\mathrm{nM} \pm \mathrm{SEM})$ & $\mathrm{Nd}$ & $\mathrm{Nd}$ & $\mathrm{Nd}$ & $887 \pm 290$ \\
$\mathrm{~A} \beta 42(\mathrm{pM} \pm \mathrm{SEM})$ & $\mathrm{Nd}$ & $\mathrm{Nd}$ & & \\
\hline
\end{tabular}

Brain A $\beta$ levels (both murine- and transgenically expressed human-species) showed that lowered neprilysin levels or expression of AD-causing human APP led to an accumulation of $\mathrm{A} \beta$ in mouse brains

Levels of $\mathrm{A} \beta 40$ and $\mathrm{A} \beta 42$ were below detection sensitivity in the formic acid fraction of WT and neprilysin-deficient mice. Moreover, $\mathrm{A} \beta 42$ was not detected in brains of WT mice, confirming previous findings that these levels are too low for detection by ELISA [51, 52]. Finally, the entire $\mathrm{A} \beta$ content in brains of the APPtg mice represented A $\beta 40$, reflecting the mild amyloid pathology in our APPtg mouse line, when compared to their NEP-APPtg littermates. The concentrations of SDS- and formic acid-soluble A $\beta 40$ in brains of NEP-APPtg mice were significantly higher than the corresponding levels in the APPtg littermates $(P<0.01$ and $<0.001$, respectively)

$N d$ not detectable 
approximately 2.7 fold higher than in the APPtg brains. Furthermore, no FA-soluble A $\beta 42$ was found in WT, NEPand APPtg brains (Table 1). As expected, neprilysin enzymatic activity was reduced in NEP- and NEP-APPtg mice when compared to their WT or APPtg littermates (Fig. 2).

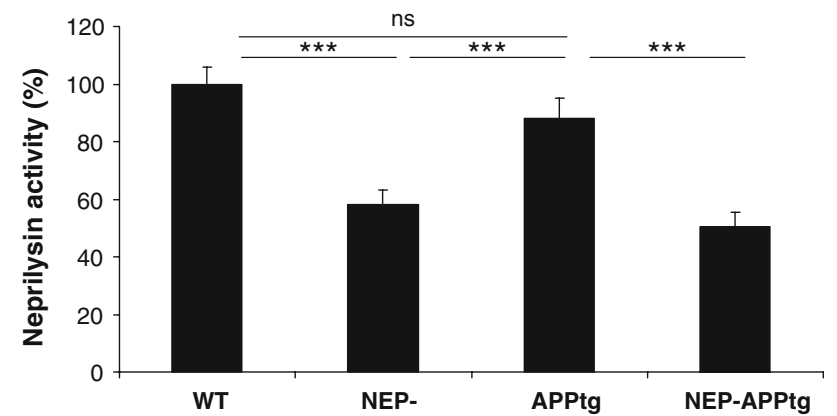

Fig. 2 Neprilysin enzymatic activity was significantly lower in NEP - and NEP-APPtg brains when compared to their WT or APPtg littermates (enzyme activity in WT brains was set at 100\%). No statistical difference was observed between the WT and APPtg groups. $n s$ not significant, $* * * P<0.0001$
In addition, no statistical difference was found in the WT and APPtg brains suggesting that over-expression of human APP did not affect brain neprilysin activity (1-way ANOVA: genotype $P<0.0001$, WT vs. NEP $-P<0.0001$, WT vs. APPtg ns, WT vs. NEP-APPtg $P<0.0001$, APPtg vs. NEPAPPtg $P<0.0001$, Fig. 2).

Neprilysin Deficiency Resulted in Worsening of Hippocampus-Dependent Cognitive Functions

In the cued navigation task, all groups learned to find the visible platform. In this task, independent of the NEP expression level, the APP transgene was associated with a small increase in escape latencies, while neprilysin deficiency itself had no effect (repeated ANOVA: APPtg+ $P<0.0055, \quad \mathrm{NEP}-\mathrm{ns}$, time $P<0.0001$, APPtg $+\times$ time ns, NEP $-\times$ time ns, NEP-APPtg ns, Fig. 3a, b). In contrast, in the place navigation task, we found that the effect of the APP transgene on escape performance depended on neprilysin deficiency (Fig. 3c, d). The presence of the APP transgene was associated with significantly
Fig. 3 APP transgene effect without $(\mathbf{a}, \mathbf{c})$ and with $(\mathbf{b}, \mathbf{d})$ concomitant neprilysin deficiency on training performance in the Morris water maze. a, b All groups learned the cue navigation task. The escape latencies (in seconds) of each two consecutive trials were calculated and the group averages were built. Neprilysin deficiency did not affect the performance in the cue navigation task, but independently of the neprilysin expression level the inheritance of APP transgene was associated with a small increase in escape latencies. (for significance levels see the "Results" section). c, d In the place navigation task, the data are suggestive of a NEP deficiency dependent effect of the APP transgene on escape performance. The escape latencies are built as above. The APP transgene was associated with significantly increased escape latencies in neprilysin deficient mice (d). In the presence of normal neprilysin levels (c) this difference was much smaller and statistically insignificant (for significance levels see the "Results" section)
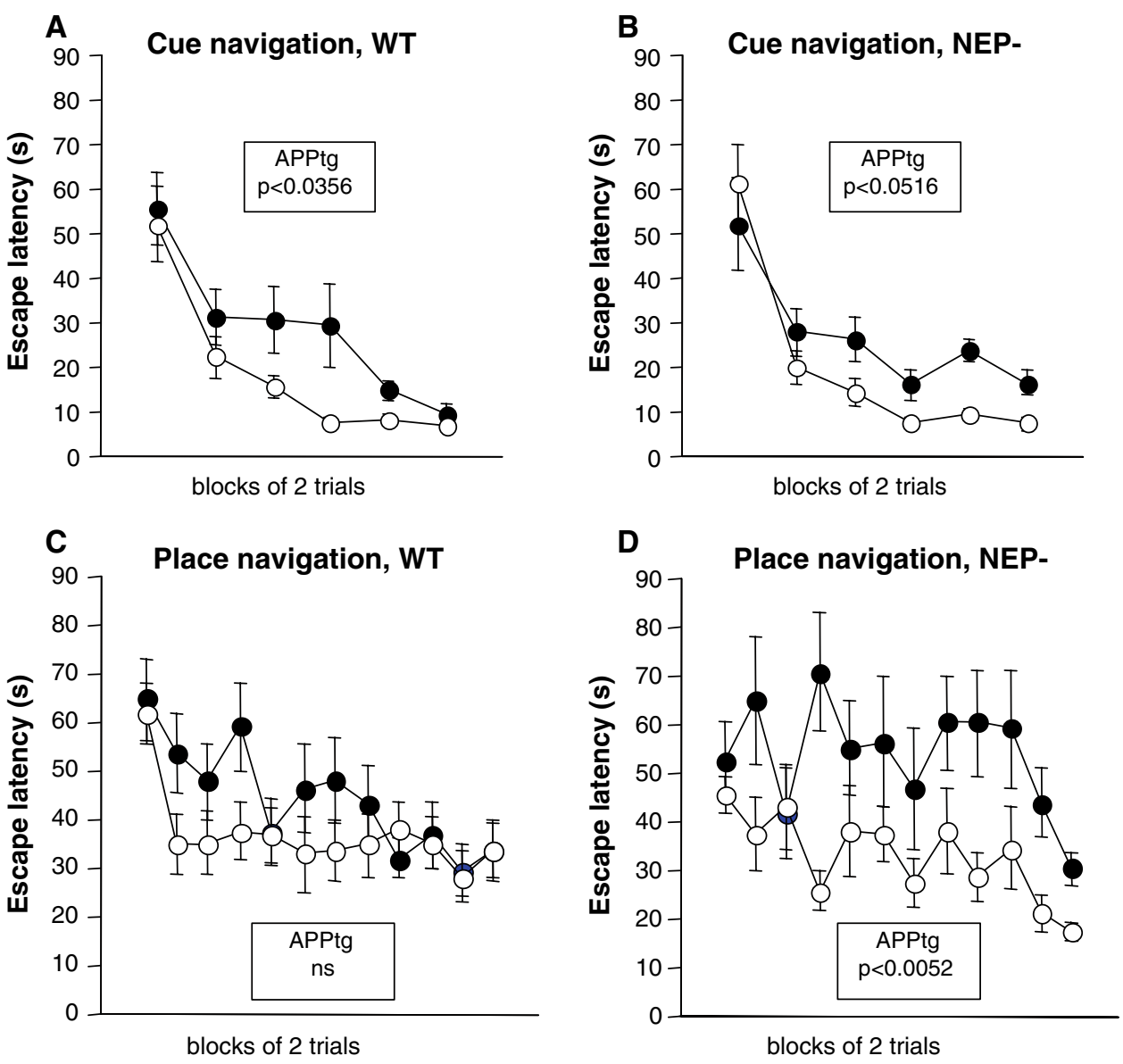
increased escape latencies in NEP- mice (Fig. 3d), while in the presence of normal neprilysin levels this difference was statistically insignificant (Fig. 3c) (repeated ANOVA: APPtg $+P<0.0023$, NEP - ns, time $P<0.0001$, APPtg $+\times$ time ns, NEP $-\times$ time $P<0.0749$, NEP-APPtg ns; split ANOVA APPtg+: in WT place ns; in NEP $-P<0.0052$, Fig. 3c, d).

The genotype affected spatial measures directly related to memory and cognition, as assessed in the probe trial (Fig. 4). Performance during the probe trial, undertaken following the MWM place navigation task, showed that all mice searched preferentially in the target zone, thus indicating successful spatial learning. Time spent in the target zone further indicated an effect of neprilysin-deficiency on spatial selectivity that was dependent on the APP transgene. The NEP-APPtg mice exhibited a reduced selectivity for the target zone when compared with their APPtg littermates (repeated ANOVA: place $P<0.0001$, APPtg $+\times$ place ns, $\mathrm{NEP}-\times$ place ns, NEP-APPtg $\times$ place $P<0.0790$; split ANOVA APPtg+: in WT place $P<0.0045$, APPtg $+\times$ place ns; in NEP - place $P<0.0057$, APPtg $+\times$ place $P<0.1068$, Fig. 4a, b). Moreover, analysis of annulus crossings, the most stringent measure of spatial selectivity, confirmed that the APP transgene was associated with a loss of spatial selectivity in the presence (Fig. 4d), but not absence of (Fig. 4c), neprilysin deficiency (repeated ANOVA: place $P<0.0031$, APPtg $+\times$ place $P<0.1076$, NEP $-\times$ place ns, NEP-APPtg $\times$ place $P<0.0228$; split ANOVA APPtg+: in WT place $P<0.0080$, AP$\mathrm{Ptg}+\times$ place ns; in NEP- place $P<0.1032$, APPtg + $\times$ place $P<0.0228$, Fig. 4c, d). APPtg mice were unimpaired in MWM testing, a result that can be explained with the mild $\mathrm{A} \beta$ pathology in our mouse line and in agreement to previous results [18]. Fig. 4 seems to suggest such an improvement in the NEP - animals. However, this trend was statistically not significant (comparison of $\mathrm{NEP}+/+$ vs. NEP - in the APPtg-: place $P<0.0079$, place $\times \mathrm{NEP}-$ $P<0.1509$ ). Probe trial measures were normalized to distance swum to avoid interference with differences in swim speed.
Fig. 4 APP transgene effect without $(\mathbf{a}, \mathbf{c})$ and with $(\mathbf{b}, \mathbf{d})$ concomitant neprilysin deficiency on performances during the probe trial. a, b The spatial selectivity was assessed by the percent of time spent in the target zone area. The APP transgene was associated with reduced selectivity in the presence (b) but not in the absence (a) of neprilysin deficiency suggesting a neprilysin-deficiency dependent APP effect (for significance levels see the "Results" section). c, d According to assessment of annulus crossings, the most stringent measure of spatial selectivity, defined by the number of crossings over the target area and respective control annuli per swam distance, the APP transgene was associated with a loss of spatial selectivity in the presence (d) but not in the absence (c) of neprilysin deficiency (for significance levels see the "Results" section)
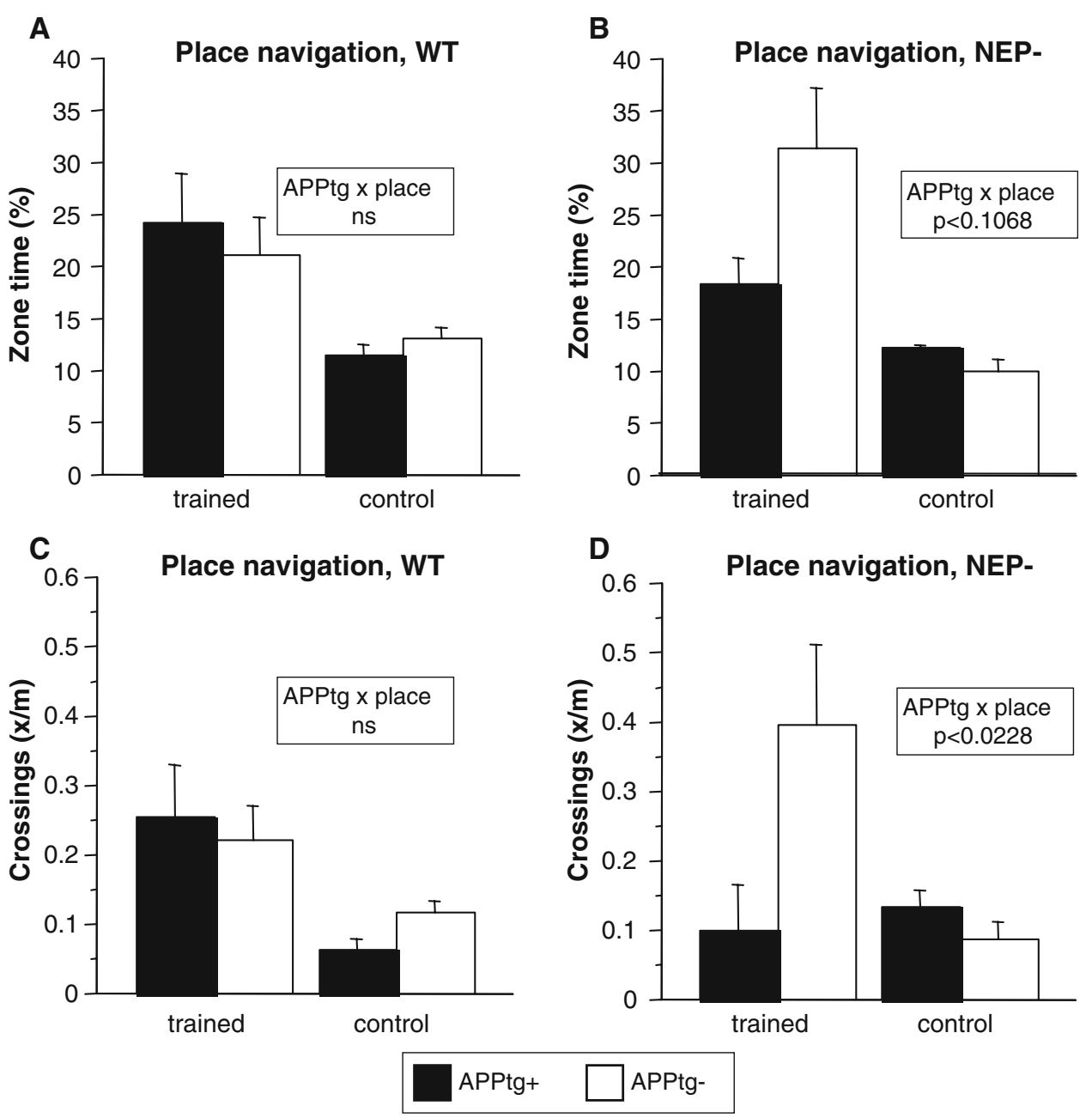
Neprilysin Deficiency Resulted in Worsening of CTA Performance

Testing mice in the CTA required subjecting mice to waterdeprivation. Therefore, to avoid any possible behavioral influences of previous training in the MWM and in order to be able to test the mice in all behavioral tests at \pm 24 months of age, we decided to use a separate mouse group for the CTA rather than using the same mice that were tested in the MWM. Age-matched mice ( $n=10$ each genotype), originating from the same parents as mice tested in the previous behavioral tests were employed to examine the hippocampus-independent associative memory in CTA paradigm.

Body weight of the mice did not vary significantly between groups during the course of the CTA test. Moreover, all groups drank comparable amounts of water during the adaptation- and conditioning-phases and genotype did not affect the amount of saccharin drunk during the conditioning trial. $48 \mathrm{~h}$ after conditioning, mice were subjected to the first choice test (Fig. 5, day 1), by presenting them with one bottle containing saccharin solution and one bottle filled with water. No differences in memory trace were found between the genotype groups in the first choice test,

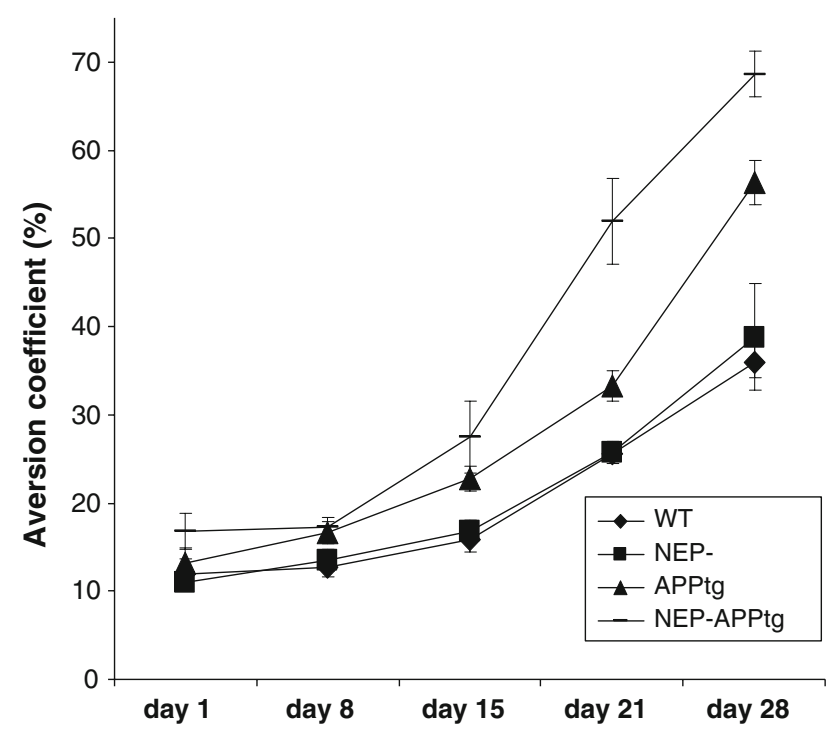

Fig. 5 Effects of APP transgene and neprilysin deficiency on memory performance in the CTA. The aversion coefficient, a measure of impairment of associative memory, defined as the percentage of saccharin consumption divided by the total fluid intake, is depicted over five choice tests. All genotype groups succeeded in CTA but saccharin intake was significantly increased in the presence of the APP transgene. Both APPtg and NEP-APPtg mice displayed a significantly diminished conditioned aversion against saccharin compared to NEP- and WT mice, with NEP-APPtg mice exhibiting the poorest memory performance. Performance of NEP mice did not differ from their WT littermates (for significance levels see the "Results" section) indicating that all groups learned the task successfully (Fig. 5, day 1). The strength of the conditioned aversion $(P<0.001)$ and the speed of its extinction were significantly different (genotype $\times$ days $P<0.001$ ) among the genotype groups (Fig. 5). The APP transgene was associated with facilitated extinction of the associative memory. During the subsequent weekly choice tests (Fig. 5, days 8, $15,21,28)$ an increase of aversion coefficient, i.e., memory impairment, was observed in all groups. WT and NEPmice displayed an equally strong aversion against saccharin, suggesting that neprilysin deficiency did not alter the CTA performance. APPtg as well as NEP-APPtg doubly mutated mice showed a significantly weaker conditioned aversion in the choice tests with NEP-APPtg showing the lowest CTA performance (ANOVA: genotype $P<0.001$, WT vs. NEP- ns, WT vs. APPtg $P<0.001$, WT vs. NEPAPPtg $P<0.001$, APPtg vs. NEP-APPtg $P<0.01)$. Taken together, these results show a negative effect of the APPtg genotype on CTA performance, which is further worsened by neprilysin deficiency.

Lastly, the avoidance of an intrinsic aversive bitter taste was determined by offering the water-deprived mice quinine solution or water. No difference between the four groups was detected $(P<0.48$, not shown), confirming that taste sensitivity did not differ between the groups.

\section{Discussion}

The findings described in this paper show that partial lack of neprilysin, resembling the lowered neprilysin levels in aged human brains, led to an enforced amyloid pathology in the brain and to impairment of both hippocampaldependent and-independent cognitive performance.

Accumulation of $\mathrm{A} \beta$ in the brain is associated with memory impairment of $\mathrm{AD}$ patients. High $\mathrm{A} \beta$ levels are shown to induce synaptic dysfunction [1, 17, 24] and to result in the formation of amyloid plaques, leading to compromised neuronal integrity and function [25-27]. The topological and spatial distribution of neprilysin suggests a physiological role in $\mathrm{A} \beta$ catabolism $[7,28]$. In addition, neprilysin is down-regulated in the hippocampus and cerebral cortex during normal aging [29, 30] and in early stages of $\mathrm{AD}$ development [10, 29, 31], thus linking an inefficient $\mathrm{A} \beta$ clearance from the brain and $\mathrm{AD}$ pathophysiology. Moreover, neprilysin has been shown to be a major peptidase capable of $\mathrm{A} \beta$ degradation in vivo [8, 9].

An important contribution to understanding the mechanisms of AD pathophysiology was achieved by generation of transgenic models over-expressing APP in brain neurons. Despite the importance of these mice in mimicking amyloid plaque pathology associated with $\mathrm{AD}$, it should be kept in mind that not only $\mathrm{A} \beta$, but also APP and APP-proteolytic 
fragments are over-expressed and thus accumulate in the brains of such models, making it difficult to assign the observed effects to $\mathrm{A} \beta$ only. This difficulty arises from the fact that APP proteolytic fragments are implicated in contradictory functions: they can be neurotoxic [32-34], may impair LTP $[35,36]$, lead to neurodegeneration [37, 38] and cognitive impairments [36, 37], while also being reported to enhance LTP [39], to be neuroprotective [40] and to up-regulate neprilysin activity [41].

A further complication arises from the fact that deviations from physiological APP level may have negative functional consequences. It was shown, in mice overexpressing APP, that $\mathrm{A} \beta$ rather than APP is involved in synaptotoxicity as measured by assessment of synaptophysin immunoreactivity [17]. Counter-intuitively, a similar decrease in synaptophysin immunoreactivity, impaired synaptic plasticity and deficits in cognitive functions were also found in mice lacking APP [42-44] and a defective memory performance was also reported in mice over-expressing WT APP [45]. These data demonstrate the complex role of APP and its photolytic fragments in neuronal function.

Reports from several laboratories, including ours, show that neprilysin degrades $\mathrm{A} \beta$ without affecting APP metabolism [13, 14, 18, 46, 47]. Therefore, in the present study, by using mice with lower neprilysin activity we were able to specifically target $\mathrm{A} \beta$ without modification of APP processing because APP processing is not changed by neprilysin deficiency [14] or over-expression [18, 46, 47]. Therefore, APP transgenic mice that also carried one defective Mme allele represent a unique experimental model to analyze effects mediated by a sole elevation of $\mathrm{A} \beta$ on cognition. The reason for employing mice with only a partial neprilysin deficiency, rather than mice with a total lack of neprilysin, was that we aimed to recapitulate the human situation as accurately as possible, in which a reduction-but not elimination — of neprilysin is detected in aged or $\mathrm{AD}$ brains. In the double-mutated mice (NEPAPPtg), the neprilysin activity was reduced to approximately half of corresponding values in APPtg brains, reflecting the reduction of neprilysin in brains of $\mathrm{AD}$ patients [48, 49]. Moreover, we employed an APPtg mouse line exhibiting a relatively mild phenotype, again to be able to model the accumulation of $\mathrm{A} \beta$ in brains of $\mathrm{AD}$ patients over an extended period of time.

Biochemical analysis revealed that in comparison with APPtg littermates, the double-mutated mice exhibited a significant decrease in neprilysin enzymatic activity and an elevation of brain $\mathrm{A} \beta$. To examine whether neprilysin deficiency affects cognitive functions, mice were tested in MWM and CTA tests, exemplifying hippocampus-dependent spatial learning and memory and hippocampusindependent associative memory, respectively.
In the open field (not shown) we found that motor and motivational characteristics were indistinguishable from each other. This finding is in concert with previous data that APPtg and neprilysin-deficient mice are normal and that the reduction of neprilysin enzymatic activity can be well tolerated by the organism $[6,16]$ and with own data that neprilysin heterozygous and homozygous mice were very similar in behavioral tests [14].

In all cognitive behavioral tests, neprilysin-deficient mice did not differ from WT controls. In the MWM test, all groups of mice were able to find the location of both a visible and a hidden platform. Moreover, NEP- mice performed similarly to their WT littermates in the probe trial, reconfirming their normal spatial memory and learning performance. In contrast, the combination of neprilysin deficiency with APP over-expression in NEP-APPtg mice was associated with behavioral impairment in the probe trial, a finding that is in line with overt accumulation of soluble and aggregated forms of $\mathrm{A} \beta$ in brains of the double-mutated animals. Therefore, inefficiency of $\mathrm{A} \beta$ removal from the brain due to a reduced neprilysin activity led to the diminished performance in the hippocampusdependent MWM.

Our data are in line with reports showing that elevated brain $\mathrm{A} \beta$ levels in mice with neuronal expression of mutated human APP or in neprilysin knock-out mice cause a worsening of CTA [14, 22]. Combining neprilysin deficiency and elevated APP expression in NEP-APPtg mice proved most destructive for CTA performance. In agreement with the MWM data, performances of WT and NEPmice in the CTA test were indistinguishable. Moreover, a negative dominant effect of the APPtg genotype on CTA performance was observed as reported earlier [18], which was accentuated in the NEP-APPtg double-mutated mice. As outlined for the MWM, these results indicate that neprilysin deficiency and the consequent accumulation of $\mathrm{A} \beta$ resulted in the inability of the double-mutated mice to perform normally in the CTA testing paradigm.

Recent data suggest that the levels of neprilysin substrates, except for $\mathrm{A} \beta$, are unchanged in brains of neprilysin knock-out mice [28], excluding the possibility that observed effects in the NEP-APPtg mice may be due to accumulation of those other neprilysin substrates. Moreover, $\mathrm{A} \beta$ degradation by neprilysin is a down-stream event after $\mathrm{A} \beta$ generation and does not affect APP metabolism $[13,14,18,46,47,50]$. These data, in combination with the fact that NEP- mice did not differ from WT littermates in their performances in the MWM and CTA behavioral tests, stress the specificity of the observed effects. They also show that a worsening of memory performance of the NEP-APPtg mice in these cognitive paradigms was caused by accumulation of $\mathrm{A} \beta$ in the brains of double-mutated mice. 
We showed here that a partial reduction of neprilysin activity and the consequent defective removal of $\mathrm{A} \beta$ from the brain led to exacerbation of amyloid pathology resulting in significant deficits of the NEP-APPtg mice in both associative and reference memory tasks when compared to their NEP - and APPtg littermates. These data are in agreement with a report showing that a complete lack of neprilysin at the site of synapses led to impaired synaptic plasticity and behavioral performance [13], and are in line with the reported selective reduction of neprilysin in sporadic $\mathrm{AD}$, particularly in brain areas with a high plaque burden [10, 11], and in normal aging [29].

Taken together, these data provide the unequivocal evidence that even a partial neprilysin insufficiency, similar to the observed reduction of neprilysin activity in aging brains, leads to an aggravation of amyloid pathology and to cognitive impairments in vivo and furthermore emphasize the importance of developing strategies to maintain physiological levels of neprilysin activity during aging and in AD therapy.

Acknowledgments We thank Drs. L. Mucke (Gladstone Institute, UCSF, CA) and B. Lu (Pulmonary Division, Children's Hospital, Boston, MA) for providing the APPtg and neprilysin deficient mice and I. Drescher, O. Litvin, J. Tracy, H. Müller and H. Li for technical help. We are also thankful to H.-P. Lipp for helpful discussions and A. Mechan for critically reading this manuscript. This work was supported by grants from the University of Zurich, NCCR Neural Plasticity and Repair, the Desirée and Niels Yde, Mobiliar and EMDO foundations.

\section{References}

1. Hardy J, Selkoe DJ (2002) The amyloid hypothesis of Alzheimer's disease: progress and problems on the road to therapeutics. Science 297:353-356. doi:10.1126/science. 1072994

2. Mohajeri MH, Saini K, Li H et al (2003) Intact spatial memory in mice with seizure-induced partial loss of hippocampal pyramidal neurons. Neurobiol Dis 12:174-181. doi:10.1016/S0969-9961(02) 00031-1

3. Reed JM, Squire LR (1997) Impaired recognition memory in patients with lesions limited to the hippocampal formation. Behav Neurosci 111:667-675. doi:10.1037/0735-7044.111.4.667

4. Rempel-Clower NL, Zola SM, Squire LR, Amaral DG (1996) Three cases of enduring memory impairment after bilateral damage limited to the hippocampal formation. J Neurosci 16 : 5233-5255

5. Tonegawa S, Tsien JZ, McHugh TJ, Huerta P, Blum KI, Wilson MA (1996) Hippocampal CA1-region-restricted knockout of NMDAR1 gene disrupts synaptic plasticity, place fields, and spatial learning. Cold Spring Harb Symp Quant Biol 61:225-238

6. Iwata N, Higuchi M, Saido TC (2005) Metabolism of amyloidbeta peptide and Alzheimer's disease. Pharmacol Ther 108:129148. doi:10.1016/j.pharmthera.2005.03.010

7. Saido TC (1998) Alzheimer's disease as proteolytic disorders: anabolism and catabolism of beta-amyloid. Neurobiol Aging 19:S69-S75. doi:10.1016/S0197-4580(98)00033-5

8. Iwata N, Tsubuki S, Takaki Y et al (2001) Metabolic regulation of brain Abeta by neprilysin. Science 292:1550-1552. doi:10.1126/ science. 1059946
9. Iwata N, Tsubuki S, Takaki Y et al (2000) Identification of the major Abeta1-42-degrading catabolic pathway in brain parenchyma: suppression leads to biochemical and pathological deposition. Nat Med 6:143-150. doi:10.1038/77399

10. Yasojima K, Akiyama H, McGeer EG, McGeer PL (2001) Reduced neprilysin in high plaque areas of Alzheimer brain: a possible relationship to deficient degradation of beta-amyloid peptide. Neurosci Lett 297:97-100. doi:10.1016/S0304-3940(00) 01675-X

11. Yasojima K, McGeer EG, McGeer PL (2001) Relationship between beta amyloid peptide generating molecules and neprilysin in Alzheimer disease and normal brain. Brain Res 919:115121. doi:10.1016/S0006-8993(01)03008-6

12. Lazarov O, Robinson J, Tang YP et al (2005) Environmental enrichment reduces Abeta levels and amyloid deposition in transgenic mice. Cell 120:701-713. doi:10.1016/j.cell.2005.01. 015

13. Huang SM, Mouri A, Kokubo H et al (2006) Neprilysin-sensitive synapse-associated Amyloid-beta peptide oligomers impair neuronal plasticity and cognitive function. J Biol Chem 281:1794117951. doi:10.1074/jbc.M601372200

14. Madani R, Poirier R, Wolfer DP et al (2006) Lack of neprilysin suffices to generate murine amyloid-like deposits in the brain and behavioral deficit in vivo. J Neurosci Res 84:1871-1878. doi: 10.1002/jnr.21074

15. Mohajeri MH, Wollmer MA, Nitsch RM (2002) Abeta 42-induced increase in neprilysin is associated with prevention of amyloid plaque formation in vivo. J Biol Chem 277:35460-35465. doi: 10.1074/jbc.M202899200

16. Lu B, Gerard NP, Kolakowski LF Jr et al (1995) Neutral endopeptidase modulation of septic shock. J Exp Med 181:2271-2275. doi:10.1084/jem.181.6.2271

17. Mucke L, Masliah E, Yu GQ et al (2000) High-level neuronal expression of abeta 1-42 in wild-type human amyloid protein precursor transgenic mice: synaptotoxicity without plaque formation. J Neurosci 20:4050-4058

18. Poirier R, WD P, Welzl $\mathrm{H}$ et al (2006) Neuronal neprilysin overexpression is associated with attenuation of $\mathrm{A} \beta$-related spatial memory deficit. Neurobiol Dis 24:475-483. doi:10.1016/ j.nbd.2006.08.003

19. Mohajeri MH, Saini K, Schultz JG, Wollmer MA, Hock C, Nitsch RM (2002) Passive immunization against beta-amyloid peptide protects central nervous system (CNS) neurons from increased vulnerability associated with an Alzheimer's disease-causing mutation. J Biol Chem 277:33012-33017. doi:10.1074/jbc.M203193200

20. Shirotani K, Tsubuki S, Iwata N et al (2001) Neprilysin degrades both amyloid beta peptides $1-40$ and 1-42 most rapidly and efficiently among thiorphan- and phosphoramidon-sensitive endopeptidases. J Biol Chem 276:21895-21901. doi:10.1074/jbc. M008511200

21. Wolfer DP, Madani R, Valenti P, Lipp HP (2001) Extended analysis of path data from mutant mice using the public domain software Wintrack. Physiol Behav 73:745-753. doi:10.1016/S0031-9384(01) 00531-5

22. Janus C, Welzl H, Hanna A et al (2004) Impaired conditioned taste aversion learning in APP transgenic mice. Neurobiol Aging 25:1213-1219. doi:10.1016/j.neurobiolaging.2003.11.007

23. Welzl H, D'Adamo P, Lipp HP (2001) Conditioned taste aversion as a learning and memory paradigm. Behav Brain Res 125:205213. doi:10.1016/S0166-4328(01)00302-3

24. Selkoe DJ (2002) Alzheimer's disease is a synaptic failure. Science 298:789-791. doi:10.1126/science.1074069

25. Brendza RP, Bacskai BJ, Cirrito JR et al (2005) Anti- A $\beta$ antibody treatment promotes the rapid recovery of amyloidassociated neuritic dystrophy in PDAPP transgenic mice. J Clin Invest 115:428-433 
26. Lombardo JA, Stern EA, McLellan ME et al (2003) Amyloidbeta antibody treatment leads to rapid normalization of plaqueinduced neuritic alterations. J Neurosci 23:10879-10883

27. Tsai J, Grutzendler J, Duff K, Gan WB (2004) Fibrillar amyloid deposition leads to local synaptic abnormalities and breakage of neuronal branches. Nat Neurosci 7:1181-1183. doi:10.1038/nn1335

28. Saido TC, Iwata N (2006) Metabolism of amyloid beta peptide and pathogenesis of Alzheimer's disease. Towards presymptomatic diagnosis, prevention and therapy. Neurosci Res 54:235-253. doi: 10.1016/j.neures.2005.12.015

29. Caccamo A, Oddo S, Sugarman MC, Akbari Y, LaFerla FM (2005) Age- and region-dependent alterations in Abeta-degrading enzymes: implications for Abeta-induced disorders. Neurobiol Aging 26:645-654. doi:10.1016/j.neurobiolaging.2004.06.013

30. Iwata N, Takaki Y, Fukami S, Tsubuki S, Saido TC (2002) Regionspecific reduction of A beta-degrading endopeptidase, neprilysin, in mouse hippocampus upon aging. J Neurosci Res 70:493-500. doi:10.1002/jnr.10390

31. Wang DS, Iwata N, Hama E, Saido TC, Dickson DW (2003) Oxidized neprilysin in aging and Alzheimer's disease brains. Biochem Biophys Res Commun 310:236-241. doi:10.1016/j.bbrc. 2003.09.003

32. Fukuchi K, Sopher B, Furlong CE, Smith AC, Dang N, Martin GM (1993) Selective neurotoxicity of COOH-terminal fragments of the beta-amyloid precursor protein. Neurosci Lett 154:145148. doi:10.1016/0304-3940(93)90192-N

33. Sopher BL, Fukuchi K, Smith AC, Leppig KA, Furlong CE, Martin GM (1994) Cytotoxicity mediated by conditional expression of a carboxyl-terminal derivative of the beta-amyloid precursor protein. Brain Res Mol Brain Res 26:207-217. doi: 10.1016/0169-328X(94)90092-2

34. Yankner BA, Dawes LR, Fisher S, Villa-Komaroff L, OsterGranite ML, Neve RL (1989) Neurotoxicity of a fragment of the amyloid precursor associated with Alzheimer's disease. Science 245:417-420. doi:10.1126/science.2474201

35. Cullen WK, Suh YH, Anwyl R, Rowan MJ (1997) Block of LTP in rat hippocampus in vivo by beta-amyloid precursor protein fragments. Neuroreport 8:3213-3217

36. Nalbantoglu J, Tirado-Santiago G, Lahsaini A et al (1997) Impaired learning and LTP in mice expressing the carboxy terminus of the Alzheimer amyloid precursor protein. Nature 387: 500-505. doi:10.1038/387500a0

37. Berger-Sweeney J, McPhie DL, Arters JA, Greenan J, OsterGranite ML, Neve RL (1999) Impairments in learning and memory accompanied by neurodegeneration in mice transgenic for the carboxyl-terminus of the amyloid precursor protein. Brain Res Mol Brain Res 66:150-162. doi:10.1016/S0169-328X(99)00014-5

38. Oster-Granite ML, McPhie DL, Greenan J, Neve RL (1996) Agedependent neuronal and synaptic degeneration in mice transgenic for the $\mathrm{C}$ terminus of the amyloid precursor protein. J Neurosci 16 : 6732-6741

39. Ishida A, Furukawa K, Keller JN, Mattson MP (1997) Secreted form of beta-amyloid precursor protein shifts the frequency dependency for induction of LTD, and enhances LTP in hippocampal slices. Neuroreport 8:2133-2137. doi:10.1097/00001756-199707070-00009

40. Mattson MP (1997) Cellular actions of beta-amyloid precursor protein and its soluble and fibrillogenic derivatives. Physiol Rev 77:1081-1132

41. Pardossi-Piquard R, Petit A, Kawarai T et al (2005) Presenilindependent transcriptional control of the Abeta-degrading enzyme neprilysin by intracellular domains of betaAPP and APLP. Neuron 46:541-554. doi:10.1016/j.neuron.2005.04.008

42. Dawson GR, Seabrook GR, Zheng H et al (1999) Age-related cognitive deficits, impaired long-term potentiation and reduction in synaptic marker density in mice lacking the beta-amyloid precursor protein. Neuroscience 90:1-13. doi:10.1016/S0306-4522(98)00 410-2

43. Seabrook GR, Smith DW, Bowery BJ et al (1999) Mechanisms contributing to the deficits in hippocampal synaptic plasticity in mice lacking amyloid precursor protein. Neuropharmacology 38:349-359. doi:10.1016/S0028-3908(98)00204-4

44. Ring S, Weyer SW, Kilian SB et al (2007) The secreted betaamyloid precursor protein ectodomain APPs alpha is sufficient to rescue the anatomical, behavioral, and electrophysiological abnormalities of APP-deficient mice. J Neurosci 27:7817-7982. doi:10.1523/JNEUROSCI.1026-07.2007

45. Moechars D, Dewachter I, Lorent K et al (1999) Early phenotypic changes in transgenic mice that overexpress different mutants of amyloid precursor protein in brain. J Biol Chem 274:6483-6492. doi:10.1074/jbc.274.10.6483

46. Howell S, Nalbantoglu J, Crine P (1995) Neutral endopeptidase can hydrolyze beta-amyloid (1-40) but shows no effect on betaamyloid precursor protein metabolism. Peptides 16:647-652. doi: 10.1016/0196-9781(95)00021-B

47. Leissring MA, Farris W, Chang AY et al (2003) Enhanced proteolysis of beta-amyloid in APP transgenic mice prevents plaque formation, secondary pathology, and premature death. Neuron 40:1087-1093. doi:10.1016/S0896-6273(03)00787-6

48. Hardy J (1997) Amyloid, the presenilins and Alzheimer's disease. Trends Neurosci 20:154-159. doi:10.1016/S0166-2236(96)01030-2

49. Selkoe DJ (1998) The cell biology of beta-amyloid precursor protein and presenilin in Alzheimer's disease. Trends Cell Biol 8:447-453. doi:10.1016/S0962-8924(98)01363-4

50. Hama E, Shirotani K, Masumoto H, Sekine-Aizawa Y, Aizawa H, Saido TC (2001) Clearance of extracellular and cell-associated amyloid beta peptide through viral expression of neprilysin in primary neurons. J Biochem 130:721-726

51. Crameri A, Biondi E, Kuehnle K et al (2006) The role of seladin-1/ DHCR24 in cholesterol biosynthesis, APP processing and Abeta generation in vivo. EMBO J 25:432-443. doi:10.1038/sj.emboj. 7600938

52. Refolo LM, Pappolla MA, LaFrancois J et al (2001) A cholesterol-lowering drug reduces beta-amyloid pathology in a transgenic mouse model of Alzheimer's disease. Neurobiol Dis 8:890-899. doi:10.1006/nbdi.2001.0422 